\title{
Lettera del Presidente
}

Carissimi Amici,

spero stiate tutti bene nonostante il periodo anomalo, precario e preoccupante che stiamo vivendo. In questi mesi di lockdown per il Covid-19, che ha segnato ognuno di noi e che segnerà per sempre la vita di ognuno di noi, noi comunque ci siamo stati sempre.

A questo proposito abbiamo attivato una survey sull'Impatto dell'epidemia Covid 19 nei pazienti affetti da malattia del rene policistico autosomico dominante (ADPKD) che si è appena conclusa. Siete stati in tantissimi a rispondere e di questo vi ringrazio. La Famiglia AIRP è veramente grandissima! I risultati verranno pubblicati nel prossimo numero di GCND.

Purtroppo, la sospensione delle attività AIRP previste per quest'anno si è prolungata a oltranza e, insieme al Consiglio Direttivo, abbiamo ritenuto che fosse arrivato il momento di proporre degli incontri di tipo diverso, utilizzando la tecnologia.

Abbiamo dovuto annullare l'Assemblea dei Soci e I'11 ${ }^{\circ}$ Workshop AIRP, che erano in programma per il 29 febbraio scorso, quando ormai tutto era organizzato: dovevamo solo prendere il treno ed arrivare a Bologna per incontrarci! Annullare è stato molto doloroso...

Come avremmo potuto stare lontani per un periodo lungo ed inimmaginabile? Come avrebbe potuto AIRP far sentire la sua presenza sempre e comunque?

In un periodo di smart working, abbiamo pensato anche noi di creare "smartAIRP, mettiamoci comodi" e di entrare nelle vostre case mentre voi state seduti sui vostri divani.
Manterremo la stessa logica adottata in precedenza per i consueti Workshop; procederemo dunque a livello regionale e verrete invitati a partecipare di volta in volta in relazione alle vostre regioni o aree geografiche di appartenenza toccandole tutte entro dicembre 2020. Intanto vi preannuncio che, dopo i primi tre incontri, il $4^{\circ}$ smartAIRP coinvolgerà la Sicilia e la Calabria il 5 settembre 2020 a partire dalle ore 15:00.

Ringrazio tutti i partecipanti di Emilia Romagna, Abruzzo, Basilicata, Molise, Sardegna, Umbria e Puglia che hanno partecipato ai primi tre smartAIRP. Abbiamo sentito la loro vicinanza nonostante la distanza e questo ci dà la forza per continuare. Grazie, grazie davvero!

Voglio infine ringraziare tutti coloro che in questi mesi, nonostante le difficoltà, ci stanno sostenendo e ci sostengono.

Vi ricordo anche che l'unione fa la forza e se tutti insieme ci diamo da fare possiamo affrontare i problemi del rene policistico nel modo migliore donando ad AIRP il vostro 5x1000 ed aiutare la ricerca scientifica sul rene policistico.

Un caro abbraccio a tutti

Luisa

Luisa Sternfeld Pavia

Presidente

AIRP - Associazione Italiana Rene Policistico

Received: June 21, 2020

Accepted: June 21, 2020

Published online: June 30, 2020

Indirizzo per la corrispondenza:

Luisa Sternfeld Pavia

AIRP Associazione Italiana Rene Policistico

Via Bazzini 2, 20131 Milano - Italia

luisa.sternfeld.airp@renepolicistico.it 